\title{
Interview with Gisbert Schneider
}

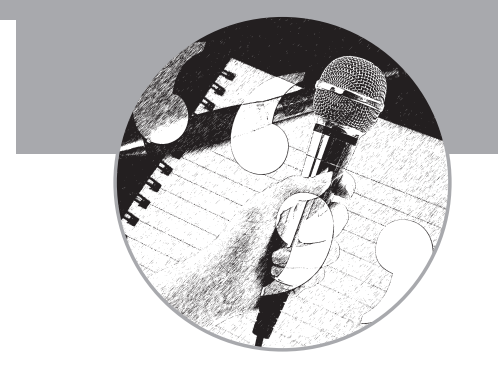

Gisbert Schneider studied biochemistry and computer science at the Free University of Berlin, Germany, where he received his doctoral degree in 1994. After several international post-doctoral research activities he joined $\mathrm{F}$. Hoffmann-La Roche Pharmaceuticals in Basel, Switzerland, where he headed the cheminformatics group until $200 \mathrm{I}$. He received his habilitation and venia legendi in biochemistry and bioinformatics from the University of Freiburg, Germany. From 2002 to 2009 he was Full Professor of Chem- and Bioinformatics (Beilstein Endowed Chair) at Goethe-University Frankfurt, Germany. In 2010 he joined ETH Zurich, Switzerland, as a Full Professor of ComputerAssisted Drug Design. Professor Schneider is the guest editor of this special focus issue on computational chemistry and has served on the editorial board since the journal launched in 2009. He spoke to Future Medicinal Chemistry about how he became involved in the field, the effects advances in software have had on research and how computational chemistry is becoming more important in the role of a traditional medicinal chemist.

Interview conducted by Isaac Bruce, Commissioning Editor.

Q At what stage in your career did you first become involved in computational medicinal chemistry and what first attracted your attention to this area?

I made 'first contact' during my graduate studies. I remember this well because I had to present a paper on neural networks in chemistry in our journal club, which, in a way, influenced my whole subsequent career path. At the time, bioinformatics was a new exciting wave in the life sciences, and I was lucky to have been able to follow these developments from the beginning. The term 'computational medicinal chemistry' is relatively new, but nicely pinpoints a part of molecular informatics that is appropriate for pharmaceutical research. The combination of developing algorithmic solutions to fundamental questions of practical relevance and realization of the idea by smart experiments in chemistry and biology still attracts and fascinates me.

Q How much computational training is required for someone with a 'traditional' medicinal chemistry background to become involved in the field? And do you think that this is often a barrier for those interested in the area?

There is no doubt that solid knowledge in computer science and informatics is a prerequisite for becoming deeply involved in this field of research. Of course, such an endeavor adds to an already fully packed chemistry curriculum. Yet, any chemist really interested to follow this track will benefit from the additional workload in the long term. The same is true for computer scientists, who would like to work in computational medicinal chemistry. Here, profound knowledge of chemistry and biology are the lacking elements to be complemented. In my view, a rock-hard education in either chemistry or computer science is a good basis. One can work from there and add the bits and pieces lacking. At the very least, prior to applying computational methods to problems in medicinal chemistry, one must understand the underlying models to be able to interpret the data generated. A good way to start is to actively include and listen to the respective other expert in practical medicinal chemistry projects.

Please can you update us on some of the research activities in which your group is currently engaged?

Our current research is both technology- and application-oriented, with a focus on the development of machine-learning models that can be used to generate new bioactive compounds, specifically drug-like agents and immunomodulatory peptides. The magic topic here is 'adaptive design' technology that combines both theoretical models and practical realization. We synthesize and biophysically characterize computationally designed compounds in our own laboratory. Peptidemembrane interactions and protein-protein interfaces are of specific current interest. Finding small compounds that modulate their structure and function is a tough nut to crack; yet this might be a particularly suitable field of research for structure-based molecular design methods.

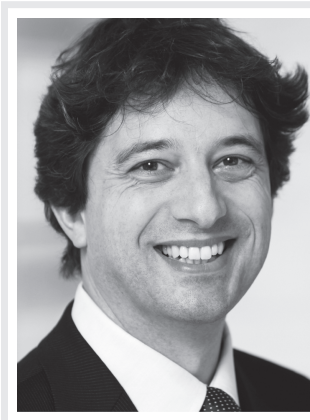

Gisbert Schneider

Swiss Federal Institute of Technology, Department of Chemistry \& Applied Biosciences, 8093 Zürich, Switzerland E-mail: gisbert.schneider@

pharma.ethz.ch 


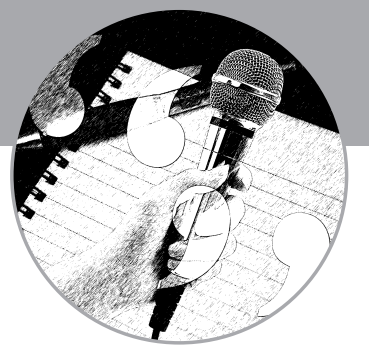

Q How has the development of improved software enabled the progression of de novo design of therapeutics? Do you feel that any initial delays in these improvements held back the field?

To the best of my knowledge, there is no therapeutic agent on the market that was computationally designed 'as is'. De novo molecular design aims at providing innovative and sometimes surprising structures to the medicinal chemist. It is not meant to come up with drugs that are ready to enter clinical trials. Without doubt, the computational techniques used for molecular design have advanced and we have learned from some of the early failures. The main hindrance has always been the lack of practical application. Often, computer-designed compounds suffer from being considered as trivial or too complex for synthesis. This is exactly the reason why our research group is dedicated to both molecular design and its practical application. I am sure that we can benefit massively from applying and playing with de novo design software.

Q You have worked in both industry and academia; how do the sectors contrast in terms of their approaches to computational medicinal chemistry?

Evidently, the industrial focus is and has to be more product oriented than research in an academic environment. As a consequence, many industrial modeling groups concentrate on most challenging applications, which require a broad spectrum of methods and expert knowledge. Academic groups enjoy the privilege of being able to develop new methods over a longer period of time than in industry and still perform proof-of-concept applications. Still, with regard to hit and lead compound identification, academic groups sometimes are over-optimistic and lack the perspective of real-world drug development.

Q Does the nature of computational research allow for international, cross-industry and industry-academia collaborations? What is your experience of this?

Sophisticated computational medicinal chemistry tools are easily distributed and publicized, maybe easier than technologically demanding 'wet' methods. In this regard, computational research is indeed ideally suited for international, cross-industry and industry-academia collaborations. There are several excellent examples that have led to mutual benefit and added value. However, there are few areas that allow for a meaningful exchange between scientists irrespective of their affiliation - without the need for data exchange. This obstacle has always hampered such collaborations, caused, in part, by legal issues and understandable reservations. In the end, it is people working together and, from my experience, this probably is the decisive factor for success.

Q In the next 5-10 years, what particular element of computational chemistry do you see advancing the most and why?

Progress in soft- and hardware technology will enable the application of ab initio methods to a much greater degree than today. This, in turn, will lead to consequently more detailed molecular representations. I expect that smart parallelization of algorithms will be a driving factor in this regard. Without doubt, during the next decade we will witness full integration of machine-learning systems in computational chemistry, a development that has already begun. Better curated databases containing standardized data on bioactive compounds, including natural products, are a necessity for better predictive models. I am curious to see progress in this area. Consequently, predictive polypharmacology and off-target prediction, automated de novo molecular design directly coupled to chemical synthesis, and focused compound library design will become routine applications in medicinal chemistry.

Q What words of encouragement would you give young scientists who might be envisioning a career in computational medicinal chemistry?

We have seen waves of huge optimism for computational medicinal chemistry followed by times of great skepticism, and renewed trust. Apparently, the field is too young or too temperamental to have found a happy medium yet. Therefore, it is mandatory to possess and maintain a frustration- and hype-tolerant character to ensure long-term satisfaction in this truly exciting and scientifically very challenging field of research (I actually feel this way). Maybe the best answer to this question is by quoting a short dialog from one of my favorite science-fiction movies: "Is it 
worth it?" "Oh yeah, it's worth it - if you're strong enough." Go for it, dig deep and keep your curiosity. There is so much yet to discover.

\section{Financial \& competing interests disclosure}

The author is a scientific consultant to pharmaceutical industry, and a co-founder of AlloCyte Pharmaceuticals. The author has no other relevant affiliations or financial involvement with any organization or entity with a financial interest in or financial conflict with the subject matter or materials discussed in the manuscript apart from those disclosed.

No writing assistance was utilized in the production of this manuscript. 